\title{
CHANGING TRENDS IN LOCAL ANESTHETIC SYSTEMIC TOXICITY AND ITS MANAGEMENT
}

Isolation of cocaine from cocoa leaves and clinical application as local anesthetic (LA) in 1884 popularised regional anesthesia (RA). ${ }^{1}$ However, by 1887 , the adverse effects of cocaine on the central nervous system (CNS) and cardiorespiratory system attributable to local anesthetic systemic toxicity (LAST) were recognised. ${ }^{2}$ Development of safer LAs in the subsequent century combined with a better understanding of mechanisms of action and manifestations of LAST, paved the way for better applications of RA and LAs. ${ }^{3}$ Changing manifestations: Keeping pace with the evolving routes of application of LAs, the appearance of LAST has also morphed. Customarily, LAST manifested prodromal features such as light headedness, perioral numbness, metallic taste, or irritability before proceeding to convulsions and, in severe cases, cardiovascular (CVS) suppression followed by cardiac arrest (CA). Current trends show increased incidence of direct cardiotoxicity, bypassing the CNS manifestations or combined manifestations of both CNS and CVS possibly due to widespread use of safer and shorter acting sedatives prior to nerve blocks to enhance patient comfort. ${ }^{3}$ Two classic presentations of LAST known earlier were development within minutes of injection

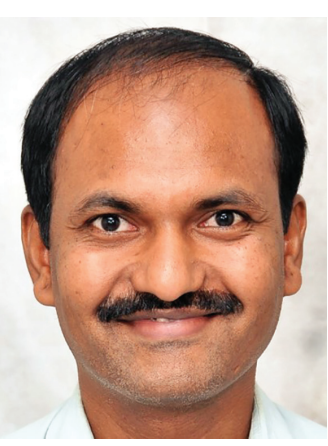
with inadvertent intravascular injection of LA or a little delayed (up to $30 \mathrm{~min}$ from injection) presentation when LA was deposited at highly vascular sites as rapid systemic absorption of the LA led to build-up of toxic plasma levels. Popularity of ultrasound-guided RA (USGRA) has opened up the floodgates for LA use in a hitherto unseen kind of way. Though the incidence of LAST due to intravascular injection has been on the downtrend with efficient use of USGRA (reduces the total dose of LA for successful block and multiple site injections can reduce the risk of significant intravascular injections), along with improvement in monitoring and development of stringent guidelines, the overall incidence of LAST has not reduced as much as may have been expected..$^{4-6}$ Development of newer modalities of blocks (especially blocks involving large quantities of LA administration), continuous catheter infusions, malfunctioning infusion pumps (excessive drug administration), accidental misconnection of LA infusion pumps to intravenous cannulae with universal Luer connectors, wrongly labelled syringes, actual body weight-based dose calculations in obese patients, have all contributed to development of LAST in the last decades.

Contemporary reports of LAST indicate a comparatively delayed presentation after initiation of RA. Popularisation of USGRA fascial plane blocks requiring deposition of large volumes of LA into fascial planes could be one factor where steady build-up of plasma concentrations, especially in patients with inherent risk factors, result in LAST manifesting as late as 60-90 min from injection time. Similarly, deposition of large quantity of LA in joints (local infiltration analgesia), wound infiltration analgesia, tumescent anesthesia, topical anesthesia and intravenous lidocaine therapy for acute and chronic pain can result in a similar presentation (even up to 2-6 $\mathrm{h}$ delayed presentations observed). Continuous catheter infusion of LA can manifest LAST as late as 24-96 h after the start of infusion, with the underlying mechanism being an imbalance in the rates of administration and elimination of the LA. ${ }^{8}$

Changes in preventing strategies: The best strategy for preventing LAST in these scenarios would be to use minimum effective dose of LA with USGRA, dose adjustment based on block site vascularity and patient risk factors (extremes of age, term gestation, severe systemic disease, patients on hepatic enzyme inhibitors or myocardial depressant medications and lean body mass-based dose for obese patients) and addition of epinephrine to LA where not contraindicated. Maintaining neutral $\mathrm{pH}$ of the blood cannot be overemphasized as alkalotic $\mathrm{pH}$ reduces the threshold for seizures while acidotic $\mathrm{pH}$ increases the free fraction of $\mathrm{LA}$ in circulation. ${ }^{4-8}$

Early detection of intravascular injection can avoid precipitation of serious LAST. Injection of intravascular markers such as epinephrine, fentanyl, even air; have been advocated for detecting intravascular placement of needle tip or catheter prior to injecting LA. Injecting LA under USG guidance constantly observing the needle tip and spread of LA can be reassuring, but safety can be enhanced by injecting LA in multiple small increments of 3-5 mL with negative aspiration for blood before each injection. Waiting for at least 30 seconds between aliquots to allow for accidental intravascular injection to reach the CNS and cause manifestations (circulation time) and increasing this duration to 45-60 seconds in patients with delayed circulation times are critical to enhance safety. ${ }^{4-8}$

Practicing preincision 'surgical pause' to discuss with colleagues of all departments involved about the risk factors and amount of LA that can be subsequently used can prevent mishaps. ${ }^{4}$ Use of LA with higher CC/CNS ratio [dose required for circulatory collapse (CC) compared with dose required for CNS manifestations]. improved vigilance during labeling and use of drugs, observing the infusion pump for at least $30 \mathrm{~min}$ after the start, preferring small-bore connectors over standard Luer lock connectors, etc. can limit the risk of LAST.4-8

Considering that nearly $50 \%$ of procedural LA administration is performed by non-anesthesiologists; ${ }^{4}$ it is imperative that they be well trained in recognition and management of LAST. The Holy Grail, expectedly, is the development of a safe LA. Liposomal-encapsulated bupivacaine $(\mathrm{LEB}){ }^{8}$ bupivacaine-impregnated collagen $(\mathrm{BIC})^{9}$ and neosaxitoxin ${ }^{10}$ are purported contenders. LEB involves nonconcentrically arranged bupivacaine molecules within liposomal capsules that result in extended release of the bupivacaine with almost $72 \mathrm{~h}$ duration of action, thus mitigating the necessity for continuous infusions and catheters. Furthermore, nonconcentric arrangement ensures that the drug is released in slow increments minimizing the build-up of plasma levels. However, until its safety profile is established, similar levels of vigilance as for other LA, should be practiced. Avoid concurrent administration of other LA with LEB as they have synergistic effects with resultant rapid peaking of plasma levels. ${ }^{4,8} \mathrm{BIC}$, a biodegradable and fully resorbable collagen matrix impregnated with bupivacaine, is an under-trial drug which acts by delivering bupivacaine directly to the site of surgical trauma thus minimizing the plasma levels. ${ }^{9}$ Neosaxitoxin, another under-trial drug has the ability to prolong anesthesia without the risk of LAST as it brings about its sensory action by binding to the outer pores of non-cardiac Na channels without crossing the blood-brain barrier. ${ }^{10}$ 
Editorial

Changes in management plans: Despite strides in safer delivery of LAs, it remains imperative to stay informed about the management of LAST. The management of prodromal manifestations remains unchanged, with immediate cessation of injection of LA and monitoring for any further effects. Convulsions should be managed first with IV benzodiazepines. Resistant convulsions can be treated with small doses of thiopentone or propofol (with small dose of succinylcholine if necessary) along with management of the airway, breathing and circulation as necessary. ${ }^{4-8}$

Development of cardiotoxic manifestations herald impending CA. Early administration of intralipid, when serious LAST (resistant seizures, arrhythmias, rapid deterioration in patient condition) is suspected, outweighs the risks related to intralipid administration which are minor and mostly self-limiting (allergy, hyperthermia, hyperlipidemia, elevation of liver enzymes, pancreatitis, etc). Only soy-based long-chain triglycerides are recommended for use. Current understanding is that the lipid shuttle mechanism has a more important role to play in mitigating the risk of LAST, where the intralipid not only absorbs the LA molecules (lipid sink/scavenge) from organs of higher perfusion (brain, heart) but also transports these to organs of lower perfusion (skeletal muscles), thus maintaining a concentration gradient that enhances the shuttling of LA from vital organs. Lipid shuttling is most effective when intralipid is administered early in the event as at that time the LA concentration is maximal at the vital organs. Moreover, the intralipid also serves as a direct energy substrate to myocardium and has cardiotonic effects by clearing LA off the myocardial cells. Furthermore, through some poorly understood mechanisms, intralipid provides beneficial postconditioning effects on recovering myocardium. ${ }^{4-8}$

The revised American Society of Regional Anesthesia (ASRA) dosages for intralipid based on patient weight should be followed. ${ }^{4}$ However, the critical point is not precise volume or flow rate but early administration of, and sustaining an infusion of intralipid in serious LAST. For resistant cardiac arrest/cardiac toxicity, the bolus doses can be repeated once or twice and infusion doses can be doubled to a maximum upper limit of $12 \mathrm{~mL} / \mathrm{kg}$ and the infusion should be continued for at least $10 \mathrm{~min}$ following cardiovascular stabilization. All patients with early resolution of LAST should be observed for at least $2 \mathrm{~h}$ and those recovering from serious LAST should be monitored for at least 4-6 h. ${ }^{4-8}$ Though anecdotal reports of successful reversal of LAST with a Chinese herbal medicine, Shenfu and glucose, insulin, potassium infusion exist, their efficacy cannot be confirmed. ${ }^{11}$

Cardiopulmonary resuscitation of LAST-induced CA has several important differences compared with conventional American Heart Association Basic Life Support and Advanced Cardiac Life Support (AHA BLS and ACLS). Hypoxia, hypercarbia and acidosis amplify myocardial-suppressant effects of LA and thus should be avoided by early airway interventions though effective chest compressions are equally important for effective lipid shuttling. Intralipid should be administered early along with airway intervention. Epinephrine in conventional doses and vasopressin increase the afterload on an already failing heart and, therefore, should be replaced by 10-100 $\mu \mathrm{g}$ epinephrine boluses with only amiodarone reserved for treatment of tachyarrhythmias. Since cardiopulmonary resuscitation may last for prolonged periods before revival, the nearest cardiopulmonary bypass unit should be alerted and the patient transferred at the earliest. ASRA checklist and ASRA LAST ${ }^{\mathrm{TM}}$ smartphone $\mathrm{app}^{12}$ serve as useful cognitive aids for facilitating correct management. Centers routinely using $\mathrm{LA}$ should stock at least $1 \mathrm{~L}$ intralipid for 30 -minute resuscitation. ${ }^{4-8}$

To conclude, safer LA drugs, improved monitoring, USG guidance for blocks and stringent guidelines have all contributed to reduction in the incidence of serious LAST. However, development of newer modalities of LA administration such as fascial plane blocks and quantity blocks have resulted in delayed development of manifestations of LAST. Better beneficial profile of intralipid in successful and meaningful reversal of LAST favors its early administration in the event of serious LAST. Prolonged vigilance and dose adjustments in atrisk patients remain vital.

Goneppanavar Umesh Professor of Anaesthesia, Department of Anesthesia DIMHANS, Dharwad

Kaur Jasvinder
Associate Professor of Anaesthesia, Department of Anesthesia DIMHANS, Dharwad

\section{REFERENCES}

1. Mattison JB. Cocaine poisoning. Med Surg Rep. 1891;115:645-650.

2. Bier AKG. Experiments in cocainization of the spinal cord, 1899. In: Faulconer A, Keys TE, trans. Foundations of Anesthesiology. Springfield, IL: Charles C Thomas; 1963:854.

3. Dickerson DM, Apfelbaum JL. Local Anesthetic Systemic Toxicity. Aesthet Surg J 2014; 34:1111-1119.

4. Neal JM, Barrington MJ, Fettiplace MR, Gitman M, Memtsoudis SG, Morwald EE, et al. The third American Society of Regional Anesthesia and Pain Medicine Practice Advisory on Local Anesthetic Systemic Toxicity. Reg Anesth Pain Med 2018;43:113-123.

5. El-Boghdadly K, Chin KJ. Local anesthetic systemic toxicity: continuing professional development. Can J Anesth 2016; 63:330-349.

6. Safety Committee of Japanese Society of Anesthesiologists. Practical guide for the management of systemic toxicity caused by local anesthetics. J Anesth 2019; 33:1-8.

7. Christie LE. Picard J, Weinberg GL. Local anaesthetic systemic toxicity. BJA Education 2015; 15:136-142.

8. El-Boghdadly K, Pawa A, Chin KJ. Local anesthetic systemic toxicity: current perspectives. Local Reg Anesth 2018; 11:35-44.

9. Cusack S, Jaros M, Kuss M, Minkowitz HS, Winkle P, Hemsen L. Clinical evaluation of Xaracoll , a bupivacaine-collagen implant, for postoperative analgesia in two multicentre, randomized, double-blind, placebo-controlled pilot studies. J Pain Res 2012; 5:217-225.

10. Rathmell JP, Strichartz G, Wanderer J. Neosaxitoxin versus traditional local anesthetics: Mechanism of action and sites of notable effect. Anesthesiology 2015; 123:A23.

11. Sekimoto K, Tobe M, Saito S. Local anesthetic toxicity: acute and chronic management. Acute Med Surg 2017; 4:152-160.

12. Neal JM, Woodward CM, Harrison TK. The American Society of Regional Anesthesia and Pain Medicine checklist for managing local anesthetic systemic toxicity. Reg Anesth Pain Med. 2018; 43:150-153. 